\title{
Some Results on the Hermite Matrix
}

\author{
Feng Zhang, Xiaoxiao Liu, Jinli Xu \\ Department of mathematics, Northeast Forestry University, Harbin, China
}

*Corresponding Author: Feng Zhang, Department of mathematics, Northeast Forestry University, Harbin, China

Abstract: This paper mainly discusses some properties of matrices under the similarity of unitary matrix. At the same time, we characterize the product of the eigenvalues of semi-positive definite matrices. Then we prove the rationality of the function operation of the Hermite matrix. Finally, we discuss the relationship between the singular values of the matrix and its sub matrix.

Keywords: Singular values; sub matrix; semi-positive definite matrix

\section{INTRODUCTION}

Proposition 1. Suppose that $A \in C_{n}$. If $A A^{*}=A^{2}$, then $A^{*}=A$.

Proof. By conditions, we know $A\left(A^{*}-A\right)=0$, and $A^{*} A^{*}=A A^{*}, A^{*}\left(A^{*}-A\right)=0$, it satisfied $\left(A-A^{*}\right)\left(A^{*}-A\right)=0$, so we prove that $A=A^{*}$. We know that any matrix is similar to the upper triangular matrix. If $A \in C_{n}$, then exists an unitary matrix $U$ such that

$$
A=U^{*}\left(\begin{array}{cccc}
a_{1} & & & \\
& a_{2} & * & \\
& & \ddots & \\
& & & a_{n}
\end{array}\right) U
$$

Thus $\prod_{j=1}^{n} a_{j}=\operatorname{det} U A U^{*}$ can be seen from the relationship between the characteristic value and determinant

As we know, the determinant is invariant under unitary similarity relation matrix. So we say $\prod_{j=1}^{n} a_{j}=\max _{U} \operatorname{det} U A U^{*}$. But we may consider a more general situation: The product of the first $k$ eigenvalues, or consider the product of the last $k$ eigenvalues. If $A$ is a positive definite Hermite matrix, then we let $A=\operatorname{diag}\left\{a_{1}, a_{2}, \ldots, a_{n}\right\}$, let $U_{0}=\left(\begin{array}{c}I_{k} \\ U_{1}\end{array}\right)$ is an unitary matrix, thus we say $\prod_{j=1}^{k} a_{j}=\operatorname{det} U_{0}{ }^{*} A U_{0}$ by calculates, therefore $\prod_{j=1}^{k} a_{j} \leq \max _{U^{*} U=I_{k}} \operatorname{det} U^{*} A U$. So if $U^{*} U=I_{k}$, we can get $U=U_{0} U_{2}$, and $U_{2}$ satisfied that $U_{2}^{*} U_{2}=I_{k}, \operatorname{det} U^{*} A U=\operatorname{det} U_{\mathrm{o}}{ }^{*} A U_{\mathrm{o}}=\prod_{j=1}^{k} a_{j}$, so we have proved that $\prod_{j=1}^{k} a_{j}=\max _{U^{*} U=I_{k}} \operatorname{det} U^{*} A U$. This is

Proposition 2. If $A$ is a positive definite Hermite matrix, then $\prod_{j=1}^{k} a_{j}=\max _{U^{*} U=I_{k}} \operatorname{det} U^{*} A U$, $\prod_{j=1}^{k} a_{n-j+1}=\min _{U^{*} U=I_{k}} \operatorname{det} U^{*} A U$ 
In addition, we can also get a conclusion like this:

Corollary. Any complex matrix are unitary similar to a matrix which diagonal elements are all equal. Proof. We use $E(i, j)$ represents a Unit matrix exchange the $i, j$ rows, Then we get $A_{i, j}=E_{i, j}{ }^{*} A E_{i, j}$, and $\sum_{i, j} A_{i, j}$ is similar to $n^{2} A$, Therefore, the conclusion holds.

Proposition 3. $A, B \in C_{n}, A$ is a positive definite Hermite matrix, $B$ is a Hermite matrix, then $A+B$ is positive if and only if $\lambda_{j}\left(A^{-1} B\right)>-1, j=1,2, \ldots, n$.

Proof. $A$ is a positive definite Hermite matrix, Then exists the inverse matrix $M$ such that $M^{*} A M=I$, as the same time, $M^{*} B M$ also is a Hermite matrix . Then exists an unitary matrix $Q$ such that $Q^{*} M^{*} B M Q=\operatorname{diag}\left\{a_{1}, a_{2}, \ldots, a_{n}\right\}, Q^{*} M^{*} A M Q=I$. Let $S=M Q$, so we can get that exists the inverse matrix $K$ such that $B=K^{*} \operatorname{diag}\left\{a_{1}, a_{2}, \ldots, a_{n}\right\} K, A=K^{*} K$, and we notice that $\left\{a_{1}, a_{2}, \ldots, a_{n}\right\} \in \operatorname{Spec}^{-1} B$, and the theory of semi-positive definite matrices shows that positive matrix only contract with positive matrix, so $A+B$ is positive if and only if $\lambda_{j}\left(A^{-1} B\right)>-1, j=1,2, \ldots, n$.

Next, we consider the relationship between the eigenvalues of the semi-positive definite matrix and the eigenvalues of its principal sub-matrix. If $A$ is a semi-positive matrix, $B$ is a sub matrix of $A$. We know $\operatorname{Spec} B \subseteq \operatorname{Spec} A$ according to $\lambda_{1}=\max _{x^{*} x=1} x^{*} A x, \lambda_{n}=\min _{x^{*} x=1} x^{*} A x, \lambda_{1}$ Represents the maximum eigenvalue of matrix $A, \lambda_{n}$ represents the minimum eigenvalue of matrix $A$. It's convenient to get $\|B\|_{2} \leq\|A\|_{2}$.

For the convenience of the next discussion, we first give two theorems:

1. Cauchy separation theorem Suppose the eigenvalues of $A \in H_{n}$ are $\lambda_{1} \geq \ldots \geq \lambda_{n}, B$ is a principal sub-matrix of order $\mathrm{m}$ for $A$, the eigenvalues of $B$ are $u_{1} \geq \ldots \geq u_{m}$. then $\lambda_{j} \geq u_{j} \geq \lambda_{n+j-m}, j=1,2, \ldots, m$.

2. Weyl monotonicity theorem Suppose $A, B \in H_{n}$, with $A \geq B$, then $\lambda_{j}(A) \geq \lambda_{j}(B), j=1,2, \ldots, n$.

Proposition 4. Let $A \in C_{n} B \in M_{r}$ is a sub-matrix of $A$, then the singular values satisfies that $s_{j}(B) \leq s_{j}(A), j=1,2, \ldots, r$.

Proof. We prove that $\lambda_{j}\left(B B^{*}\right) \leq \lambda_{j}\left(A A^{*}\right)$, generally, we may assume that $A=\left(\begin{array}{cc}B & D \\ C & M\end{array}\right)$, we get $\lambda_{j}\left(B B^{*}+D D^{*}\right) \leq \lambda_{j}\left(A A^{*}\right)$ by Cauchy separation theorem, we get $\lambda_{j}\left(B B^{*}\right) \leq \lambda_{j}\left(B B^{*}+D D^{*}\right)$ by Weyl monotonicity theorem, so we prove that $\lambda_{j}\left(B B^{*}\right) \leq \lambda_{j}\left(B B^{*}+D D^{*}\right) \leq \lambda_{j}\left(A A^{*}\right)$.

Corollary 4. Let $A, B$ are two matrices, then $\lambda_{j}\left(A^{2}+B^{2}\right) \leq \delta_{j}\left(\begin{array}{cc}A & B \\ B & A\end{array}\right)$, where $\lambda_{j}$ represents the $j-t h$ eigenvalue of the matrix, $\delta_{j}$ represents the $j-t h$ singular value of the matrix.

Corollary 5 : The modulus of the characteristic root of any sub-matrix of the unitary matrix is less than or equal to1.

We don't use Weierstrass theorem to prove. The function operation of the Hermite matrix is independent of the specific spectral decomposition. We define the function operation of Hermite matrix:

Proposition 6. Let $f(t)$ is a real-valued continuous function on $\Omega$, the eigenvalues of Hermite matrix $H$ include in $\Omega, H=\operatorname{Udiag}\left(\lambda_{1}, \ldots, \lambda_{n}\right) U^{*}$ is the spectral decomposition of $H, U$ is an unitary matrix, then definition of the function operation of Hermite matrix $H$ is 


$$
f(H)=\operatorname{Udiag}\left(f\left(\lambda_{1}\right), \ldots, f\left(\lambda_{n}\right)\right) U^{*} .
$$

Proof. Let $H=\operatorname{Udiag}\left(\lambda_{1}, \ldots, \lambda_{n}\right) U^{*}, H=\operatorname{Vdiag}\left(\lambda_{1}, \ldots, \lambda_{n}\right) V^{*}, U, V$ are unitary matrices, Hence $U=V\left(\begin{array}{llll}A_{1} & & & \\ & A_{2} & & \\ & & \ddots & \\ & & & A_{r}\end{array}\right)$, and $A_{i} A_{i}^{*}=A_{i}^{*} A_{i}=I$, so we get

$\operatorname{Udiag}\left(f\left(\lambda_{1}\right), \ldots, f\left(\lambda_{n}\right)\right) U^{*}=\operatorname{Vdiag}\left(f\left(\lambda_{1}\right), \ldots, f\left(\lambda_{n}\right)\right) V^{*}$ by directcalculates.

The above theorem helps to study the partial order of the matrix. The following proposition is to consider the variation of the eigenvalues of the diagonal matrix under the function of the unitary matrix.

Proposition 6 If $U$ is an unitary matrix with $n-t h$ order, and $A=\operatorname{diag}\left(a_{1}, a_{2}, \ldots, a_{n}\right), a_{i}$ are all real numbers, then the eigenvalues of $U A$ are $\lambda_{j}$, and it holds that:

$m \leq\left|\lambda_{j}\right| \leq M, m=\min _{j} a_{j}, M=\max _{j} a_{j}$.

Proof. We can prove $\left|\lambda_{j}\right| \leq M$ by $\delta_{\max } U A=\delta_{\max } A$, using the same way, we can prove $m \leq\left|\lambda_{j}\right|$, so $m \leq\left|\lambda_{j}\right| \leq M$.

From the relationship between the eigenvalues of the matrix and the determinant, the following proposition can be obtained.

Proposition 7 Let is $A$ a semi-positive definite real symmetric matrix with $n$-th order, $E \geq A$. Then it holds that: we have $\operatorname{det}(E-A K) \geq \operatorname{det}(E-A)$ for any orthogonal matrix $K$.

Proof. We get $\operatorname{det}(E-A O) \geq 0$ by $E-A \geq 0$ and Proposition 6 , If one of the eigenvalues of $A$ is1, Proposition has already been proved. So we consider the case where the eigenvalues of $A$ are all less than 1 .At this time, Proposition can be proved by the condition that the positive definite matrix can be contracted simultaneously to the diagonal matrix. And we can see that the necessary and sufficient condition for the equation to be established is $K=E$.

\section{REFERENCES}

[1] Grone R, Johnson C R, Sá E M, et al. Positive definite completions of partial Hermitian matrices[J]. Linear Algebra \& Its Applications, 1984, 58(APR):109-124.

[2] Chekhov L, Eynard B. Hermitian matrix model free energy: Feynman graph technique for all genera[J]. Journal of High Energy Physics, 2005, 15(3):014.

[3] Akemann G. Higher genus correlators for the hermitian matrix model with multiple cuts[J]. Nuclear Physics B, 1996, 482(1-2):403-430.

[4] Wenbao A I, Huang Y, Zhang S. New results on Hermitian matrix rank-one decomposition[J]. Mathematical Programming, 2011, 128(1-2):253-283.

[5] Tian Y. Equalities and inequalities for inertias of hermitian matrices with applications[J]. Linear Algebra \& Its Applications, 2010, 433(1):263-296.

Citation: F.Zhang.et.al, (2019). Some Results on the Hermite Matrix. International Journal of Scientific and Innovative Mathematical Research (IJSIMR), 7(1), pp.14-16 http://dx.doi.org/10.20431/2347-3142. 0701003

Copyright: (c) 2019 Authors, This is an open-access article distributed under the terms of the Creative Commons Attribution License, which permits unrestricted use, distribution, and reproduction in any medium, provided the original author and source are credited. 